\title{
La "Fuga a quatro todas las bozes Por vna Sexto tono" de Antonio de Cabezón y su textualidad parcial en Melchior Franck (Da pacem Domine)
}

\author{
The "Fuga a Quatro todas las bozes por VNa SEXto tono" by ANTonio de Cabezón \\ and its partial textualisation by Melchior Franck (DA PaCem Domine)
}

\author{
Stephan Schmitt \\ Hochschule für Musik und Theater, München
}

\begin{abstract}
Resumen
La fuga a quatro de Antonio de Cabezón es un canon a cuatro voces y una de las piezas más extraordinarias de la colección Obras de música para tecla, arpa y vihuela... debido a su compleja estructura. La señal característica consiste en la interdependencia parcial de los sujetos. Al lado de eso, se puede entrever un principio de variación que señala hacia un futuro lejano: la variación "en evolución". El canon Da pacem Domine (popular en el área lingüística alemana) de Melchior Franck es una textualización del comienzo de la Fuga a quatro de Cabezón, sin que el compositor Franck hubiera indicado este origen.
\end{abstract}

\section{Palabras clave}

Antonio de Cabezón; Tomás de Santa María; Melchior Franck; fuga; fuga a quatro; canon; tiento; análisis; Da pacem Domine.

La fuga a quatro (vol. III, p. 73 ss.; Vol. IV, p. 44 ss. y edición facsímil, fol. $84 \mathrm{r}-84 \mathrm{v})^{1}$ representa una de las piezas más extraordinarias de la colección y debe ser objeto de una investigación más minuciosa por su excepcional habilidad artística y compleja estructura, todo lo cual, sin embargo,

1 En la edición de Anglés, aparece esta pieza como número LXX también en el vol. III, p. 28 ss. En la tabulatura abarca las páginas $84 \mathrm{r}$ y v. En el prólogo (p. 12), Anglés se refiere al viaje de Cabezón a Londres del año 1554, en el que pudo haber conocido a Thomas Tallis y "la técnica practicada en el presente tiento". No entiendo, sin embargo, la noticia editorial sobre la fuga en la misma página, pues no veo discrepancia alguna con relación al compás 21 entre la edición y la tabulatura.

\begin{abstract}
The fuga a quatro by Antonio de Cabezón is a four-voice canon. It is one of the most extraordinary pieces of the collection Obras de música para tecla, arpa y vihuela... because of its complex structure. Its characteristic feature lies in the partial interdependence of the subjects. In addition, we can glimpse a principle of variation which foresees a distant future: The continuously developing variation. The canon Da pacem Domine of Melchior Franck (well-known in German speaking countries) is a textualisation of the beginning of Cabezón's Fuga a quatro, yet without any indication of this origin on the part of the composer Franck.
\end{abstract}

Key words

Antonio de Cabezón; Tomás de Santa María; Melchior Franck; fugue; fuga a quatro; canon; tiento; analysis; Da pacem Domine.

no se revela tan fácilmente ${ }^{2}$. Que el virtuosismo técnicocompositivo, unido a la armoniosa sonoridad eran valorados también fuera de España, lo demuestra la recepción y "textualización" de los dos primeros sujetos (soggetti) en Melchior Frank, algo no revelado hasta el presente.

En esta Fuga a quatro, se trata de un canon a cuatro voces, como indica la frase añadida todas las boces por vna, que el término fuga también daba a entender en aquella épo-

2 Que yo sepa, la pieza (fuga) ha sido citada hasta hoy por FROTSCHER, 1935: 255. HOWELL 1967: 393 (nota 13). EDLER 1997: 323. 
$\mathrm{ca}^{3}$. Las cuatro voces están agrupadas, en efecto, por medio de una serie de distancias temporales y espaciales, organizadas como suma de dos canones a dos voces a la octava ${ }^{4}$. Las entradas, dentro de los canones a dos voces, se suceden rápidamente unas a otras (fuga ad semibrevem) a distancia de cuarta inferior (canon ad hypodiatessaron); entre ambos pares de voces hay una distancia de dos semibreves, es decir, de dos compases.

Vale la pena discutir el problema de si la fuga puede todavía ser denominada como tiento. Anglés, en la primera edición completa, la denomina como Tiento XIII; los Cabezón, sin embargo, la titulan como Fuga a quatro todas las bozes por vna sexto tono. Edler (p. 322 s.), que aún no conocía la nueva edición completa, acepta la denominación tiento, pero califica esta pieza como un caso límite. Tomás de Santa María, en el Libro llamado arte de tañer fantasía, capítulo 33 Del modo de hazer fugas, escribe: "Para esto es de notar, que solas dos maneras diferentes se hallan de tañer a duo. La vna se haze en fuga, y la otra sin fuga, de las quales la mas perfecta y de mas arte y primor, es la que se haze en fuga..." (p. 64 r) Por lo tanto, respecto a los dúos compuestos imitativamente, distingue entre fuga (= canon) e imitación no-canónica. Bajo el nombre de Fantasía, que aparece en el título de su tratado, dedicado a la improvisación y composición de tientos, subsume también la fuga, es decir, el canon. Llama la atención, sin embargo, que los Cabezón renuncien, precisamente aquí, al término tiento.

En la respuesta real ${ }^{5}$, que distingue a un canon, Cabezón da mucha importancia a la rigurosa igualdad interváli-

3 Cabezón, en efecto, usa todavía el significado original del término fuga, aunque que ya, desde finales del siglo XV, también se denominaban con ese término las formas imitativas no-canónicas. Cabezón usa el término otras veces, pero no para referirse a una forma, sino sólo a una técnica de composición. Una vez, se usa como término adicional en el Tiento del 3. tono fugas al contrario (vol. II: 154 ss.). Aquí, en algunos momentos de la composición contrapuntístico-imitativa, dos voces forman un breve canon por movimiento contrario. Otra de las veces, dicho término aparece en el canto llano elaborado a cinco voces Ad Dominum cum tribularer. Fuga en 4. con el tiple (vol. IV: 205 ss.), donde las dos voces superiores se mueven consecutivamente en forma de canon. Además, en el Dvo [II] (vol. I: 60 s.), aparece un canon, que abarca los primeros 17 compases, aunque sin ser denominado con dicho término.

4 Una semejante correspondencia a pares en la composición a cuatro voces es descrita también por Bermudo en su Declaración (ver también FROTSCHER, 1935: 250) y por Tomás de Santa María en el Arte de tañer fantasía, donde dicho autor, en los ejemplos de la p. 64 r s. como Cabezón en su fuga, hace comenzar las voces en forma de fragmentos de escala estrechados y, muchas veces, a distancia de cuarta inferior (KINKELDEY, 1910: $51 \mathrm{~s}$.).

5 En la única diferencia existente en el compás 90 (en el tenor semibrevis en vez de mínima), se trata ciertamente de un descuido, en que el autor de la tabulatura ha olvidado un signo de pausa. Las demás voces tienen una mínima al final del sujeto 14. La corrección no produce problemas técnico-compositivos y queda justificada por la rigurosa disposición de toda la pieza. ca, algo que raramente sucede en el canon interválico. Pero con una excepción: la mayoría de las veces se evita el VII. grado (mi); pero cuando aparece, la respuesta es a la cuarta inferior, si bemol, en vez de si (S 6: T. 34 ss. und S 8: 40 ss. respectivamente, S 6 und S 8) ${ }^{6}$. Sería imaginable, copiar el canon en su totalidad como una voz única, añadiendo las

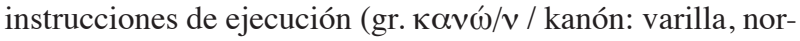
ma, regla), es decir, cómo debe componerse el canon por las cuatro voces. También habría que fijar la citada rebaja de medio tono del VII. grado en las entradas a la cuarta inferior. Pero dificilmente encontraremos un instrumentista de tecla o cuerda con una capacidad imaginativa tan marcadamente polifónica, que estuviera en condiciones de tocar la pieza entera partiendo de esa apuntación. Cabezón, sin embargo, como organista ciego era capaz de hacerlo 7 . Con esto, persiste el modo abreviado de escribir un canon, que ha llevado al concepto de forma, sólo lleno de sentido cuando es ejecutado por músicos solistas, es decir, por ejecutantes de instrumentos melódicos o cantantes.

El sexto tono con la nota fundamental en fa y con el IV. grado rebajado medio tono, caracteriza toda la composición, pero a la vez pone de relieve un sorprendente, aunque sólo aparente, arranque de la tonalidad. Esta camina desde el compás 20 a través de las cuatro voces, se dirige hacia la disonancia y falsa relación (c. 21 s. fa sostenido en el alto / fa natural en el bajo) y justifica pasajeramente el tono si del cuarto grado (es decir: fa sostenido en la cuarta inferior), que, en el hipolidio, está separado de la nota fundamental por un intervalo de trítono.

Una audición superficial de las obras de Cabezón permite percibir únicamente el fluir tranquilo y poco espectacular de formas aparentemente amorfas, interrumpido a lo sumo por pocas, aunque inesperadas, extravagancias harmónicas. En el presente caso, esta impresión está aún reforzada por la falta de cláusulas propias, que es el resultado, por una parte, de la estructura canónica per se, donde una misma melodía camina a través de todas las cuatro voces. La pequeña distancia de una semibreve entre las entradas impide, por otra parte, que los destacados movimientos cadenciales, situados en los sujetos, puedan surtir efecto. Una atenta mirada a la partitura y un escuchar preciso revelan una variedad asombrosa de detalles y una voluntad de forma, que somete el microcosmos de una configuración total y une la "abundancia épica" de pormenores a un desarrollo no sólo suficiente sino necesario. Los sujetos son - salvo pocas excepciones - abarcables en su extensión (la mayoría de las

6 En adelante: sujeto $=\mathrm{S}$

7 Respecto al problema de la ceguera y sus consecuencias para la idea musical véase también SCHMITT 2011: $121 \mathrm{~s}$. 
veces de cuatro a seis compases) y están separados unos de otros por pausas, que constituyen también el central medio de articulación del canon. Así es posible, si se escucha atentamente, percibir el caminar de las entradas de arriba hacia abajo y el cruce con los siguientes sujetos y, a la vez, observar el cambio sutil de los sonidos.

El ejemplo 1 ofrece los 16 sujetos distintos, identificados, para simplificar, por sus limitaciones de pausas, aunque, de este modo, aparecen sujetos cortísimos (S 6 y S 7) que podrían unirse con el precedente o el siguiente, o tambien muy largos (S 14 y S 15). El último sujeto (S 16) se repite y, así termina de un modo relevante. Los sujetos se mueven en la mayoría de los casos dentro del ámbito reducido de una cuarta o una quinta. Aparecen las siguientes excepciones: $\mathrm{S}$ 6 (tercera menor), S 8 (octava) y S 14 (séptima menor). Una señal característica, descrita ya reiteradamente en relación con las obras de Cabezón, aparece aquí como característica principal: la interdependencia parcial de los sujetos ${ }^{8}$. Sustancialmente pueden reducirse a dos módulos: a. un fragmento de escala en el marco de una quarta o quinta (ascendente o descendente: a o a'), b. una figura de tres miembros en saltos (quarta descendente, tercera ascendente), como pueden verse en la primera línea del ejemplo 1. El empleo casi permanente de estos módulos en forma inalterada o solo un poco modificada (véase el subrayado de los grupos de notas) produce la impresión de gran calma y homogeneidad. El sujeto final repetido (S 16/17) se presenta incluso como combinación (invertida) de los S 9 y S 10 que aparecen en el centro del canon, por lo que también el S 10 es casi idéntico al S 2 y el S 9 abarca totalmente al S 1. Incluso en el S 13, no subrayado aquí, se pueden descubrir conexiones con el módulo b. Por medio de la inmersión, rica en variantes, en el ambiente melódico y de la diversidad de sonidos resultantes se impide toda monotonía. El parentesco de los S 1 hasta el 4 , sin embargo, no se explica suficientemente, construyen- do su arquetipo en el módulo a. Detalles de la construcción muestran más bien una relación de estos sujetos con el anterior respectivamente, de modo que los S 3 y S 4 no solo se muestran como variantes del S 1 , sino que se derivan de sus directos predecesores. Con esto, se puede entrever un principio de variación que señala hacia un futuro lejano: la variación "en evolución”, tal como la describe Schönberg en el ejemplo de Brahms.

En el ejemplo 2 se muestran otras relaciones, en cierto modo, no-temáticas, porque no se derivan de los dos módulos a y b. La sinopsis de las dos tablas muestra el parentesco de los sujetos aislados en su multiformidad. El S 1 está presente en los siguientes S 2 hasta el S 4 en forma progresivamente variada y desemboca en el movimiento de tres notas del módulo b, que lleva su propia vida y aparece aislado como S 7 . Los destacados descensos de quinta al comienzo de los $\mathrm{S} 2$ y 3 cierran el S 8 en combinación. El S 6 es y el S 11 contiene una cláusula de soprano, que recorre consecuentemente por todas las voces respectivamente. A la tendencia ascendente del S 1 se contrapone, en el S 9, con la inversión del módulo a, un movimiento descendente en reposadas semibreves, que anticipa el final del canon y reduce el tempo interno, de lo que resulta una división en dos partes de todo el canon. En la segunda parte, saltan a la vista dos sujetos largos, que insinúan una articulación interna por repetición (S 14), o bien, por secuencia (S 15). Después, el último sujeto como combinación de los sujetos centrales $\mathrm{S} 10$ (que abarca los S 2 y S 1) y S 9 cierra el canon. Del tranquilo caminar del módulo a en movimiento ascendente y descendente surge por consiguiente un arco melódico. El microcosmos de relaciones entre cada una de las figuras melódicas y el desarrollo en totalidad nunca son previsibles, sino irregulares. De ahí que la pieza, a pesar de su construcción ejecutivo-inprovisatoria actúe como un sondeo de posibilidades, para conseguir con un material reducido una gran abundancia de formas. 


\section{Ejemplo 1}
a
b
$b_{0} \cdot 0(a)$

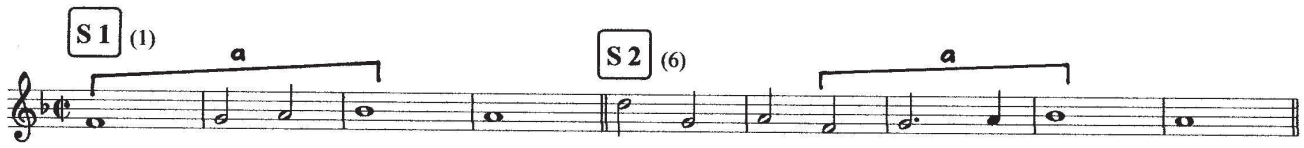

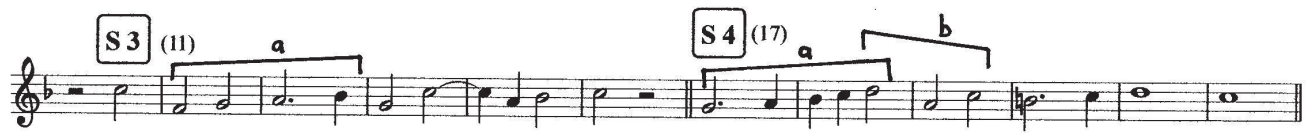

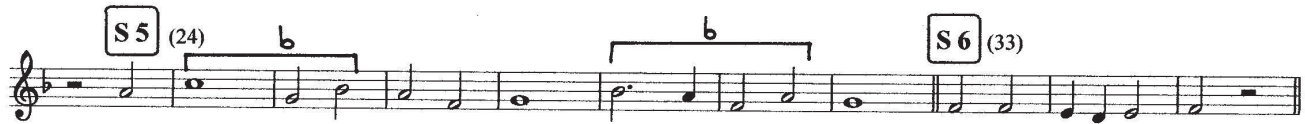

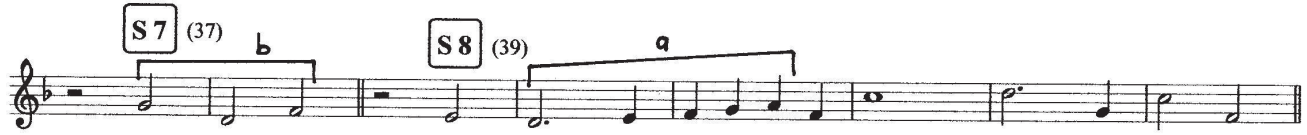

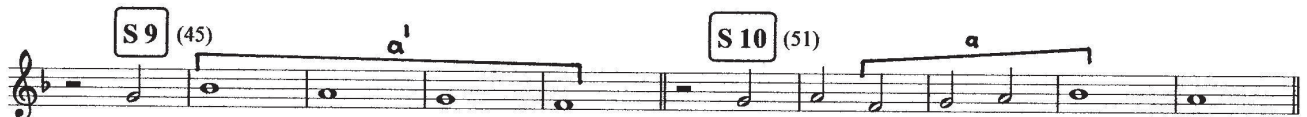

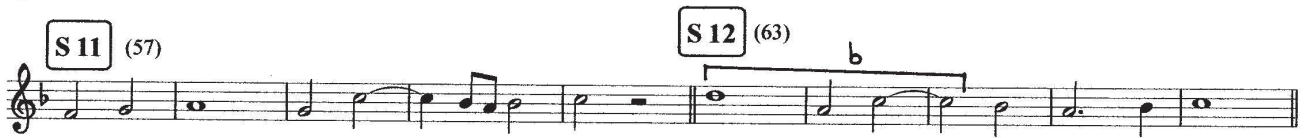
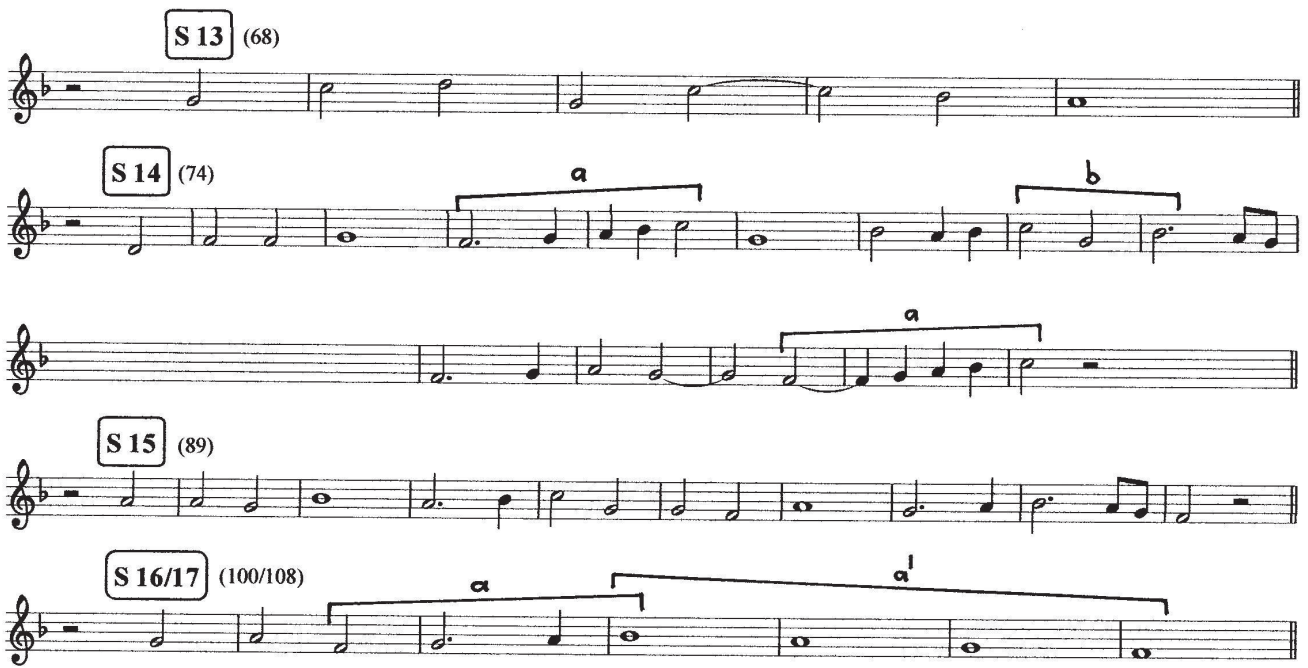


\section{Ejemplo 2}
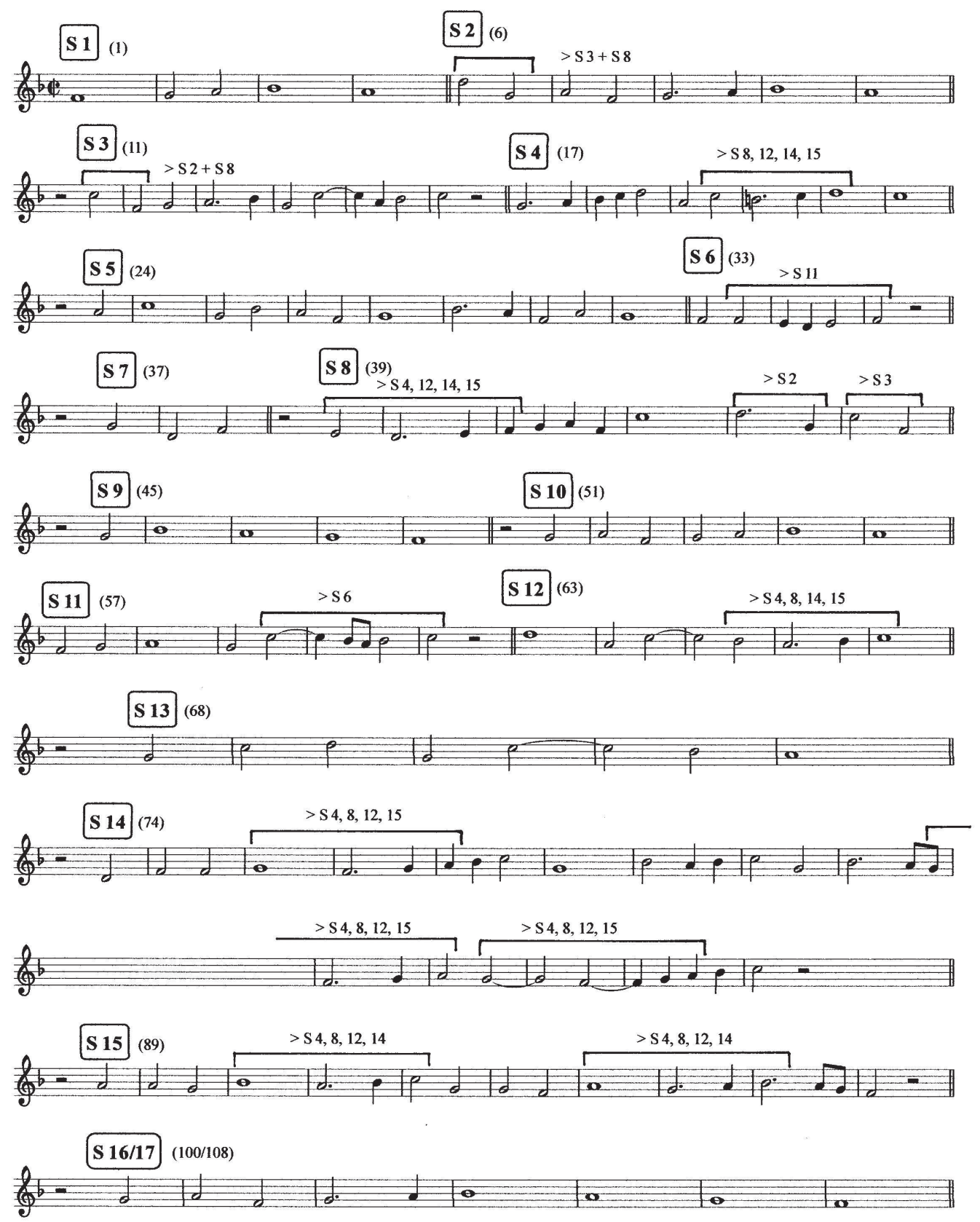
En el canon, la forma de los sujetos determina definitiva y exclusivamente la disposición sonora, ya que no es posible influir por respuesta tonal, continuación o incluso complementos no-imitativos, como acaso en el tiento o su equivalente italiano, el ricercare. Cada nota de la composición es temática, porque las respuestas son idénticas al motivo (dux). En el caso presente los sujetos son semejantes entre si, porque son reducibles a módulos comunes o proceden unos de otros. La muy confirmada homogeneidad dentro de la diversidad puede asimismo llevar a la monotonía, si la sucesión de las harmonías resultantes no produce un cambio.

Cabezón soluciona el problema, haciendo predominar el sonido central fa y la quinta do (a veces, también la cuarta si bemol) al principio (S $1+\mathrm{S} 2$ ), en dos lugares de la primera mitad (S 6 + S 9) y al final (S 16/17), para producir y asegurar la orientación tonal. Entre tanto, las demás notas del sexto modo encuentran su sitio. Esto no llamaría necesariamente la atención, si no se encontrasen en amplios trechos al principio de los compases sólo acordes invertidos o incluso disonancias (tt. 6-12, 14-21, 59-66, 68-76, 85-91). Junto a la ya citada, condicionada por la estructura, escasez de cláusulas, que crean cesuras claras, aparece una sonoridad flotante, que siempre empuja hacia adelante $\mathrm{y}$, solo al final, llega inesperadamente al reposo.

El tratamiento de las disonancias puede ser completamente intrincado, como se ve en las mínimas de los S 3 y S 4 en la composición a cuatro voces (tt. 15/16 + 17). La tensión sonora al comienzo del compás no se resuelve precisamente en el sonido esperado, sino que, en cierto modo, salta por encima de esa nota y alcanza así el sonido siguiente (t. 15 sonido de resolución esperado: si bemol, sonido alcanzado realmente: sol; t. 16 re en vez de fa; t. 18 sol en vez de si bemol). De un modo realmente estridente actúa la desviación armónica en el compás 20 ss., que ya es perceptible en la única alteración de un sujeto en toda la fuga: en el S 4 es resuelto el si bemol. El acorde aumentado sobre re (c. 21), que surge por esto, no es realmente insólito en Cabezón. La posición cercana de los acordes "fa mayor" y "mi menor" subraya el pasaje dentro del discurso harmónico, determinado por el IV. grado rebajado, si bemol, mientras enmarca la estridente disonancia del compás 21 , es decir, la prepara y la resuelve. El lugar paralelo al acorde aumentado, donde se desarrolla el segundo par de voces del S 4, prescinde ya del si bemol, con lo que se suaviza el compás 24.

La coincidencia, varias veces descrita, de rasgos arcaicos y "modernos" en la música de Cabezón ${ }^{9}$ puede observarse en efecto también en la Fuga a quatro, quizás de un modo más conciso, que en otras composiciones. Un canon, como forma más rigurosa de construcción musical, corre siempre el peligro de descuidar la sonoridad, sobre todo si es muy extenso. Precisamente en el campo de lo sonoro, Cabezón produce algo ciertamente asombroso, cuando enmarca las arcáicas progresiones sonoras con elementos funcionales como la sucesión Fa-Do, y cuando hace converger tendencias arcaicas y avanzadas en una expresiva sonoridad tensa, pues la sucesión de "acordes" $\mathrm{Fa}-\mathrm{mi}-\mathrm{Re}^{+}-\mathrm{Fa}-\mathrm{mi}-\mathrm{Re}$ - la - sol señala tanto hacia atrás como hacia adelante (c. 20 ss.).

El comienzo a dos voces del canon muestra una clara coincidencia con un ejemplo práctico del capítulo ya citado sobre la fuga en Santa María (p. 64 r, véase ejemplo 3). Esto no sorprende por dos motivos: Primero, porque Cabezón conocía la obra teórica publicada en 1565, pues el emitió un dictamen sobre dicho libro. Alguna pieza de las Obras podría haber sido concebida como un auténtico botón de muestra para lo dicho por Santa María y allí solo esbozado en ejemplos musicales ${ }^{10}$ o como dice Kastner "[...] su teoría de composición ofrece en algún aspecto un comentario sobre las Obras de Cabezón"11. En segundo lugar, porque en un fragmento de escala ascendente se trata de una forma tan elemental, que puede encontrarse en compositores de todos los tiempos, incluso sin conocerse mutuamente ${ }^{12}$.

9 APEL, 1967: 125 y 187. Véase algo semejante también en Cabanilles: SCHMITT, 2001.

10 Véase también KINKELDEY: "Ejercicios como los mencionados [i. e. ejercicios de contrapunto en Arte de tañer... de Santa María] se encuentran escritos en las Obras de Cabezón.” p. 108.

11 MGG XI (1963): columna 1379.

12 Véase el tema de la primera fuga (Do mayor) en El clave bien temperado I, de J. S. Bach. 


\section{Ejemplo 3}

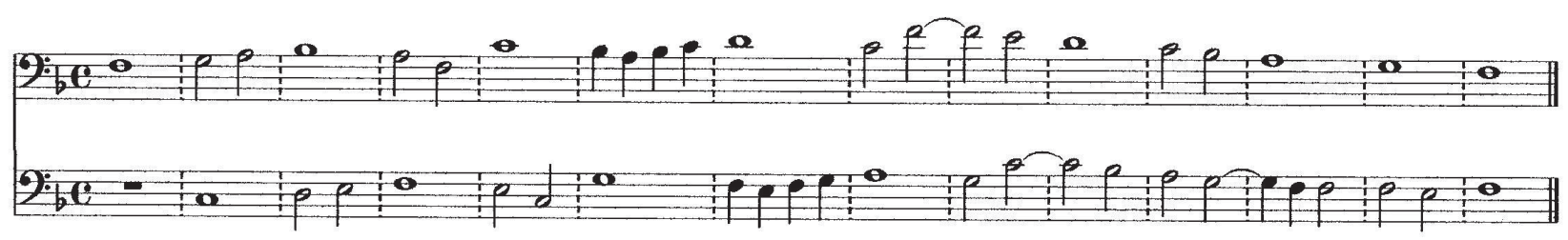

\section{MELCHIOR FRANCK: $D_{A}$ PACEM DOMINE}

En Melchior Franck (1579-1639) aparece su popularidad durante la vida y la difusión de su extensa obra de por lo menos 1500 composiciones en contraste con la escasa atención que se le presta en la actualidad ${ }^{13}$. No existe aún una edición completa de sus obras; una gran parte no ha sido aún editada. Tampoco hay información sobre su procedencia, ni existe ningún retrato suyo y, hasta hoy, sólo se conoce un renglón de notas escrito por su mano, cuyo original ha desaparecido.

Este renglón (Ejemplo 4 en el facsímil) ${ }^{14}$ reproduce uno de los cánones con texto religioso más conocidos dentro del área de habla alemana ${ }^{15}$.

El catálogo de obras de Aufdemberge (p. 229) lo muestra con la nota siguiente:

\section{$1629^{7}$}

Fuga 4. Vo: Da pacem Domine in diebus nostris. Clarissimo Excellentiss: Viro, Dno Johanni Friderico Weißio, Med: Doctorj, in suj memoriam... haec inserebat Coburgi 2 Septem: 1629 Melchior Francus.

(Canon a la cuarta inferior a 4 voces.)

(Nota escrita a mano de Franck en el álbum de visitantes de un médico de Coburgo, que antiguamente

\section{Gramß.}

14 MGG IV (1955): columna 665.

15 He aquí una selección: Gottfried WOLTERS (ed.): Ars Musica, IV Chorbuch für gemischte Stimmen, Wolfenbüttel 1965: 268. Josef HEER y otros: Musik im Leben, Ausgabe für Bayern, München 1973: 194. - Verband Bayerischer Musikerzieher (ed.): Lied \& Song, München 1976: 73. - Karl-Jürgen KEMMELMAYER/Rudolf NYKRIN (ed.): Spielpläne Musik 9/10, Stuttgart, 1988: 44. - Walter LAYHER: Lieder ohne Grenzen, München ${ }^{2}$ 1991: 80. - Bernhard BINKOWSKI y otros (ed.): Canto. Unser Liederbuch, Hannover 1996: 152. - (sin ed.): Das Kanon-Buch. 400 Kanons aus 8 Jahrhunderten zu allen Gelegenheiten, Mainz, 1999: 304. - Klaus BRECHT / Stefan KALMER (ed.): unisono, Das Liederbuch, Leipzig 2005: 232. se encontraba en Weimar, pero ahora parece perdida. Véase edición en facsímil en MGG, Vol IV, col. 665)

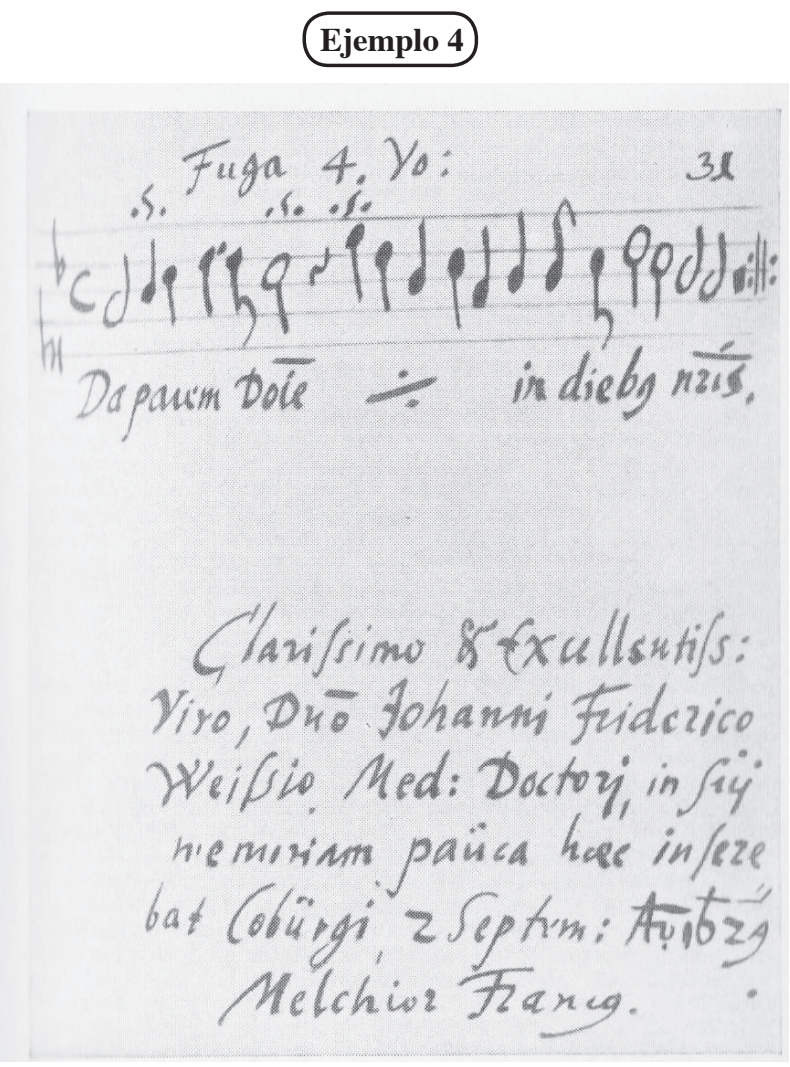

Este canon Da pacem Domine, que Franck escribió en el álbum del médico y presunto amigo Dr. Johann Friedrich Weiße, es sin duda alguna una textualización del comienzo de la Fuga a quatro de Cabezón. Franck hace uso de los $\mathrm{S} 1$ y S 2, reduciendo a la mitad el valor de las notas (Ejemplo 5). Con lo cual, completa el $\mathrm{S} 2$ con el apoyo en el final del $\mathrm{S}$ 9 (o sea $S$ 16/17), creando con ello un canon circular (canon ad perpetuum). La aplicación del texto "Da pacem Domi- 
ne, in diebus nostris" ${ }^{16}$ de la antífona gregoriana ${ }^{17}$ exige una anacrusa antes de la segunda parte del texto; esta es la única modificación melódica: al principio del segundo sujeto se antepone un do, seguido de si bemol en vez de re. La sucesión de entradas corresponde a la Fuga a quatro y, con esto, el canon de Franck (ejemplo 6) ${ }^{18}$ es idéntico a la Fuga de Cabezón hasta la citada adaptación con el inicio (tt. 1-10) y el final (tt. 113-114).
Con esto se plantean dos preguntas: 1. ¿De qué conocía Franck la Fuga a quatro de Cabezón? y 2. ¿Por qué Franck no dio a conocer la procedencia de la cita? Hoy es dificil contestar ambas preguntas ${ }^{19}$. Composiciones ocasionales breves, como las incluidas en un álbum, seguro que solo raras veces adquieren la categoría de obras más extensas, destinadas a determinados eventos y a la publicación. Por otro lado, el destinatario puede esperar, que la relación

\section{Ejemplo 5}

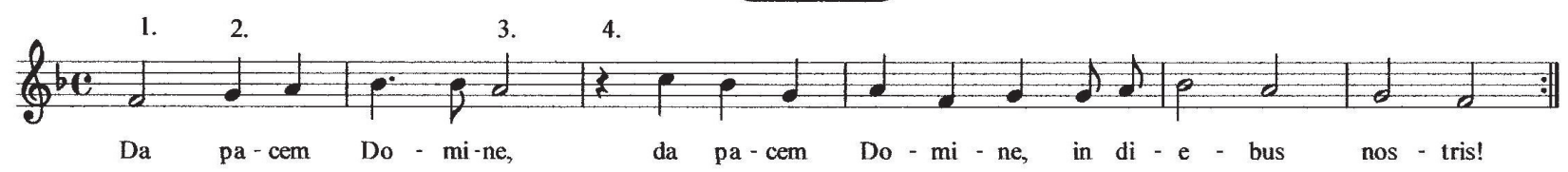

Ejemplo 6

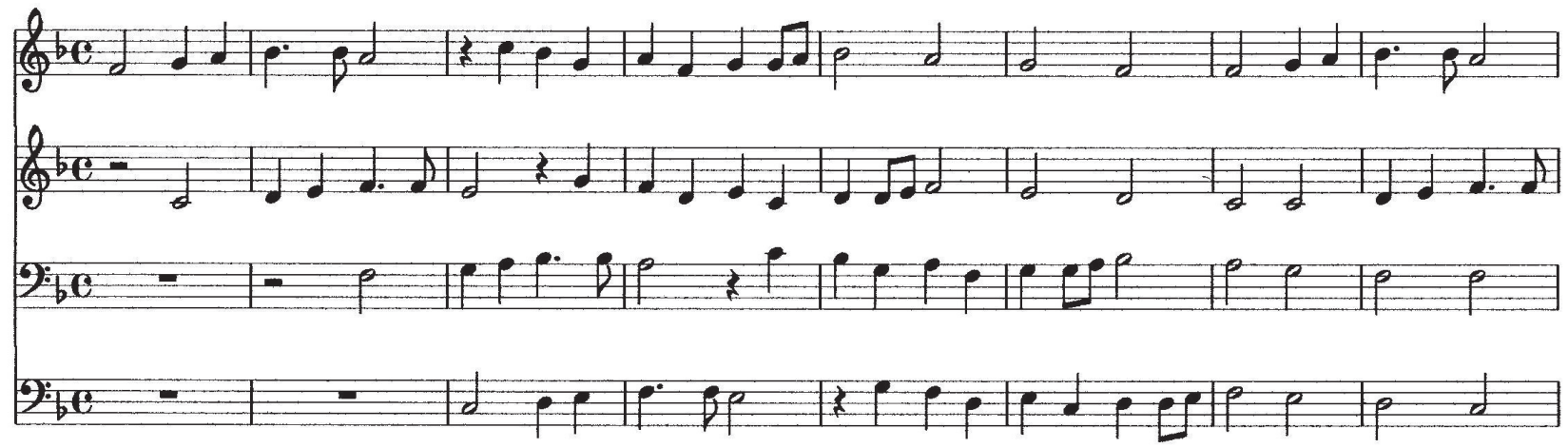

16 Franck ya había compuesto el texto completo en el nr. 32 de su Viridarium Musicum 1613, si bien sin relación musical con el canon.

17 Antiphonale Monasticum (Solesmes 2005), ad lib. Ant. para el Magnificat de las primeras vísperas del domingo XXXII del año en el Leccionario II.

18 Las subdivisiones necesarias por la aplicación del texto en los compases 2 y 4 no alteran la textura.
19 Franck, nacido en Zittau/Silesia, según lo que se sabe, vivió después la mayor parte del tiempo en Augsburgo y Coburg (Baviera). No se sabe si tuvo acceso a un ejemplar de las Obras. Existen varios ejemplares según RISM, uno en Wolfenbüttel y otro en la Deutsche Staatsbibliothek de Berlín. Otro ejemplar se encuentra según RISM (tomo suplementario) en la Bischöfliche Zentralbibliothek Regensburg. La demanda de información ha dado por resultado que el ejemplar de Wolfenbüttel no haya sido catalogado hasta 1659. El ejemplar de Berlín no llegó a la biblioteca antes de 1851. En Regensburg, a pesar de la información del RISM, las Obras no han sido catalogadas, ni existen. 
personal entre él y el escritor induzca a éste último a insertar también algo de caracter personal, es decir, algo propio. La firma de un compositor debajo de la inscripión ha sido siempre la garantía, de que presentaba una composición propia o una cita de sus propias obras ${ }^{20}$.

La reelaboración por medio de la aplicación de un texto, a principios del siglo XVII, pudo haber sido un caso marginal legal. No siempre se hizo patente la procedencia de la borrowed music ("música prestada"). Pero ya, en el renacimiento, se había formado una consciencia de los derechos de la personalidad, si bien no se desarrollaría hasta mucho más tarde la necesidad de codificar los derechos de explotación económica ${ }^{21}$. La posibilidad teórica, de que la sustancia musical del canon Da pacem Domine no proceda ni de Franck ni de Cabezón, sino de otro compositor, de que Cabezón lo haya usado como cabeza de su Fuga a quatro y Franck le haya aplicado un texto, no puede desde luego descartarse. Las características de la composición sin embargo remiten claramente a Antonio de Cabezón. Fuera del modo que fuese la práctica de adopción en tiempos del barroco temprano - y se trata de mucho más que una mera cita - es un agravio contra Antonio de Cabezón, que otra persona se apropie de su música y su éxito. Queda la esperanza de que, en nuestros tiempos, muy sensibilizados con los problemas de plagio y derechos de autor, no sigua ya poniéndose en las nuevas ediciones del canon religioso: "Texto y música: Melchior Franck (1629)", sino los datos correctos: "Aplicación del texto: Melchior Franck (1629), música: Antonio de Cabezón (antes de 1578)".

\section{BIBLIOGRAFÍA}

APEL, Willi, Geschichte der Orgel- und Klaviermusik bis 1700, Kassel, Bärenreiter, 1967.

AUFDEMBERGE, Clarence Theodore, "Vollständiges Werkverzeichnis der Kompositionen von Melchior Franck", Jb. der Coburger Landesstiftung, Coburg, Landesstiftung, 1975: 187-240.
CABEZÓN, Antonio de, Obras de música para tecla, arpa y vihuela..., Madrid, 1578, ed. Felipe Pedrell, nueva edición, ed. Higinio Anglés, Barcelona, CSIC, 1966.

EDLER, Arnfried, "Gattungen der Musik für Tasteninstrumente", Teil 1: Von den Anfängen bis 1750, Handbuch der musikalischen Gattungen, ed. Siegfried Mauser, Laaber, Laaber, 1997.

FROTSCHER, Gotthold, Geschichte des Orgelspiels und der Orgelkomposition, Berlin-Schöneberg, Hesse, 1935.

GRAMSS, Knut, "Der schicksalsreiche Weg des Werkes Melchior Francks als lebendiges Kapitel Musikgeschichte", Jb. der Coburger Landesstiftung, Coburg, Landesstiftung, 1975: 179-186.

HOWELL, Almonte C., "Cabezón: An Essay in Structural Analysis", Musical Quarterly, 50 (Oxford Univ. Press, 1964): 18-30.

HOWELL, Almonte C., "Paired Imitation in 16th Century Spanish Keyboard Music", Musical Quarterly, 54 (Oxford Univ. Press, 1967): 377.

ITOH, Tatsuhiko, Music and Musicians in the German Stammbücher from ca. 1750 to ca. 1815, Ann Arbor/Michigan, UMI,1991.

KINKELDEY, Otto, Orgel und Klavier in der Musik des 16. Jahrhunderts, Leipzig, Breitkopf \& Härtel, 1910.

ROIG-FRANCOLÍ, Miguel Angel, "Figura y obras de Tomás de Santa María”, Revista de musicología, 15 (Madrid 1992): 55-85.

SANTA MARÍA, Tomás de, Libro llamado arte de tañer fantasía. Valladolid 1565. Edición facsímil, ed. Luis Antonio González Marín, Barcelona, CSIC, 2007.

SCHMITT, Stephan, "Antonio Cabezón y el comienzo de la música didáctica para tecla con valor artístico", Аnиario musical, 66 (Barcelona 2011): 119-136.

SCHMITT, Stephan, "Vexierklänge. Anmerkungen zu einem Tiento de falsas von Juan Cabanilles", Anuario musical, 56 (Barcelona 2001): 115-130.

Recibido: 29.10 .2012

Aceptado: 29.10 .2012

20 Véase los muchos ejemplos en el trabajo de Itoh.

21 Thomas BÖSCHE: "Urheberrecht", $M G G$ neu, Sachteil, vol. 9: columna 1203 ss. 